\title{
Are there risks associated with using mindfulness in the treatment of psychopathology?
}

\author{
"...both the classical meditation literature and empirical research findings appear \\ to concur that when correctly practiced and administered, mindfulness meditation \\ is a safe, noninvasive and cost-effective instrument for improving psychological \\ health..."
}

Keywords: Buddhism $\bullet$ meditation $\bullet$ mindfulness $\bullet$ psychopathology $\bullet$ psychotic episodes

\begin{abstract}
Are there risks associated with using mindfulness in the treatment of psychopathology?

Mindfulness is a form of meditation that derives from Buddhist practice. It is the process of engaging a full, direct and active awareness of experienced phenomena that is spiritual in aspect and that is maintained from one moment to the next. Mindfulness is arguably one of the fastest growing areas of psychological empirical inquiry. For example, approximately 600 scientific papers were published on the topic in 2013 compared with just 50 scientific papers concerning mindfulness published in 2003 [1]. Over $70 \%$ of general practitioners in the UK now believe that mindfulness/meditation can be beneficial for patients with psychiatric disorders, and $20 \%$ of UK general practitioners are currently able to access mindfulness treatments for their patients [2].
\end{abstract}

Although interest and research into the utility of mindfulness-based interventions (MBIs) has significantly increased in recent years, the only psychiatric disorder for which both the UK National Institute for Health and Care Excellence and the American Psychiatric Association currently advocate mindfulness as a therapeutic intervention is recurrent depression in adults [1]. Despite this, MBIs are presently used as techniques for treating and/or improving a wide range of mental health issues within multiple applied settings (e.g., educational, developmental, forensic, clinical, occupational, mili- tary, medical, etc.) [3]. Recently, the present authors argued that the rapidity at which mindfulness was being operationalized within applied psychological domains may compromise the long-term efficacy and credibility of MBIs as well as the need to ensure that they are safely administered to serviceusers [4]. Based on empirical research findings and established practice guidelines outlined in the traditional meditation literature, it is timely to discuss and evaluate whether there are risks associated with utilizing MBIs as psychopathology treatments.

\section{Adverse effects of mindfulness: the empirical research literature}

In the research literature, reports of adverse effects relating to mindfulness meditation are uncommon. However, the scope of any form of empirical inquiry - whether quantitative or qualitative - is governed by the hypothesis for which the study is designed to test. For example, randomized controlled trial designs are frequently employed in MBI efficacy studies yet some authors consider randomized controlled trials to be reductionist due to evaluating only a limited number of predefined outcomes (and thus disregarding participants' wider experiences) [5]. Similarly, although there is a growing body of qualitative research that has attempted to analyze participants' wider experiences of practicing mindfulness, such literature is arguably underdeveloped and the majority of MBI qualitative studies tend to focus on a limited

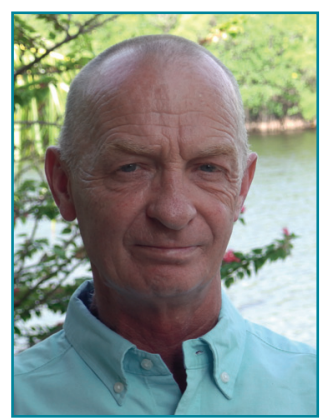

\section{Edo Shonin}

Author for correspondence: Division of Psychology, Chaucer Building Nottingham Trent University, Burton Street, Nottingham, NG1 4BU, UK

Tel.: +4401158484460 meditation@ntu.ac.uk

William Van Gordon Division of Psychology, Chaucer Building, Nottingham Trent University, Burton Street, Nottingham, NG1 4BU, UK

Mark D Griffiths

Division of Psychology, Chaucer Building, Nottingham Trent University, Burton Street, Nottingham, NG1 4BU, UK

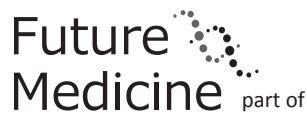


range of psychopathologies (i.e., anxiety disorders and mood disorders). Furthermore, qualitative findings are also heavily governed and/or restricted by the type of questions and technique (e.g., a semi-structured interview) used for eliciting participant responses - and such questions may be influenced by the researcher's epistemological stance.

Given that robust empirical studies explicitly investigating whether MBI participation can incur adverse effects have yet to be undertaken, the eventuality of MBIs leading to nonsalutary health outcomes remains a possibility. This possibility is made even more real by the fact that: adverse effects have been observed for other (i.e., nonmindfulness) forms of meditation [6,7]; there are detailed accounts in the classical Buddhist meditation literature of how meditation and/or mindfulness practice can detrimentally impact upon psychosocial (and spiritual) functioning [3]; and in the opinion of the present authors, studies yielding flat or negative effects can often experience difficulty in being accepted for publication.

\section{"Recent meta-analyses indicate that mindfulness-based interventions can be efficacious psychopathology treatments for mood and anxiety disorders."}

Reports of meditation-induced adverse effects in the empirical literature are invariably associated with meditation techniques such as Transcendental Meditation, Qigong and nonspecific relaxation-based meditative approaches. It is important to establish that although different meditative modes share some common attributes (e.g., introspection, relaxation, concentration, etc.), they invariably reflect fundamentally different theoretical and practical approaches. For example, Transcendental Meditation is a Hindu-derived commercial technique that was introduced in the 1950s by Maharishi Mahesh Yogi and includes mantra recitation [8]. Conversely, mindfulness meditation is a 2500 -yearold Buddhist practice that does not include chanting or mantra recitation but principally focuses on present-moment awareness [8]. Nevertheless, in meditative interventions that are not explicitly mindfulness-based, there is often a deficiency of detail regarding the exact composition of the meditative approach and it is not uncommon for such interventions to integrate techniques (e.g., breath awareness) that are also employed in MBIs [8]. Thus, although in these cases it is difficult to attribute any interventional outcomes (positive or negative) to mindfulness practices, it is certainly feasible that such practices exert a mechanistic effect.

Documented adverse effects for techniques such as Transcendental Meditation and Qigong include (but are not restricted to) panic attacks, antisocial behavior, impaired reality testing, dissociation, musculoskeletal pain, uncomfortable kinesthetic sensations, suicidal feelings and exhaustion [6]. A recent literature review carried out by the present authors identified six separate clinical case studies (12 participants in total) in which psychotic episodes were precipitated by participation in mindfulness-encompassing (but not exclusively mindfulness-based) meditative modes [8]. In the majority of instances, the case study participants had a history of mental illness (and in some cases psychotic episodes) but at least two of these case study participants were without a history of psychiatric illness [9].

There are also reports in the empirical research literature of individuals becoming addicted to meditation. These include cases of meditation addiction with negative consequences (e.g., psychological distress, dissociation, interpersonal conflict, etc.) [6] and a single case of an individual developing a 'positive' addiction to meditation [10]. In the latter instance, it was reported that a female with co-occurring schizophrenia and pathological gambling that received an 8-week intervention known as Meditation Awareness Training was successfully treated by substituting her maladaptive gambling behavior with a routine of daily-meditation practice (the intervention employed mindfulness exercises in conjunction with other meditative modes).

Finally, several recent studies by the present authors have exposed the extent to which MBI participants place emphasis on the meditative experience and competency of MBI instructors [10,11]. Qualitative findings indicate that participants respond more favorably to the therapeutic intervention when they feel the mindfulness instructor is able to impart an authentic-embodied transmission of the mindfulness teachings [11]. This is a particularly important point because there is growing concern among both academicians and clinicians that some MBI instructors may lack the necessary grounding in this respect. For example, although there are currently attempts to disseminate best-practice and assessment guidelines for some MBI teachers [12], as yet, there are no dedicated regulation and accreditation bodies to stipulate minimum competency levels for $\mathrm{MBI}$ instructors [3]. Indeed, MBI instructors can have as little as 12 months' mindfulness practice and teaching experience following completion of a single 8 -week course $[2,3]$.

\section{Adverse effects of mindfulness: the classical meditation literature}

Outside of the empirical and clinical literature, there exist cautionary teachings and/or reports in the traditional Buddhist literature of how incorrect meditation and/or mindfulness practice can lead to deleterious 
health outcomes. Although they have not been empirically established, some of these guidelines can be up to 2500 years old and are accepted as valid by many Buddhist scholars and practitioners based on a 'tried and tested' methodology. Examples of such outcomes include: asociality; nihilistic and/or defeatist outlooks; dependency on 'meditative bliss'; engaging in compassionate activity beyond one's spiritual capacity (and at the expense of psychological well-being); dysphoric mood states due to trapped 'spiritual wind' - a possible consequence of forced/incorrect breathing during mindfulness/meditative practice; and spiritual addiction (a form of self-deception in which rather than potentiating spiritual development and subduing selfish or egotistical tendencies, meditation practice becomes addictive and serves only to increase ego attachment and narcissistic behavior) [3,13-15]. Another possible risk of mindfulness practice intimated in the Buddhist teachings is that of MBI participants and instructors becoming 'too mindful'. In many respects, this relates to the aforementioned possible adverse outcome of spiritual addiction and essentially refers to a personality affectation whereby an individual makes excessive efforts to master the art of appearing to 'be mindful' (e.g., adopting an overly-pious demeanor, constant/inappropriate smiling, etc.) without actually having any presence of mind.

\section{Conclusion}

As noted above, there has been a marked growth of interest, empirical inquiry and utilization of MBIs. Recent meta-analyses indicate that MBIs can be efficacious psychopathology treatments for mood and anxiety disorders $[16,17]$. Preliminary evidence also suggests that MBIs may be effective treatments for (among other things) addiction disorders (both chemical [e.g., substance addiction] and behavioral [e.g., gambling addiction]), anger dysregulation, schizophrenia-spectrum disorders, pain disorders (e.g., fibromyalgia), attention deficit hyperactivity disorder and sexual dysfunction $[1,4]$. However, due to the rapidity at which MBIs are being clinically

\section{References}

1 Shonin E, Van Gordon W, Griffiths MD. Medication as meditation: are attitudes changing? Br. J. Gen. Pract. 63, 654 (2013).

2 Mental Health Foundation. Be mindful report (2010). www.mentalhealth.org.uk/publications/be-mindful-report

3 Shonin E, Van Gordon W, Griffiths MD. The emerging role of Buddhism in clinical psychology: towards effective integration. Psychol. Relig. Spiritual. 6, 123-137 (2014).

4 Shonin E, Van Gordon W, Griffiths, MD. Mindfulnessbased interventions: towards mindful clinical integration. Front. Psychol. 4, 194 (2013). operationalized, and the tendency for novel interventional approaches to sometimes be engulfed by a degree of hype (originating from clinicians/academicians and service users alike), there is a paucity of robust research that specifically assesses whether MBIs can induce nonsalutatory health outcomes. Furthermore, in addition to cautionary guidelines in the traditional Buddhist literature elucidating the risks of incorrectly-practiced meditation/mindfulness, there are also a number of instances where meditation - including some meditative modes that appear to employ mindfulness techniques - has precipitated deleterious health consequences (e.g., psychotic episodes, meditation addiction, antisocial and/or asocial behavior) [8].

Notwithstanding this, both the classical meditation literature and empirical research findings appear to concur that when correctly practiced and administered, mindfulness meditation is a safe, noninvasive and costeffective instrument for improving psychological health among a wide range of different groups $[3,18]$. Indeed, most of the risks related to MBIs appear to arise from a poor understanding by some academicians and clinicians as to what exactly constitutes effective mindfulness practice, as well as how to impart an authentic embodied transmission of the mindfulness teachings $[3-4,19]$. MBIs are likely to increasingly feature in the agendas of mental health stakeholders, but it would be prudent to set time aside to consolidate research findings, explore potential adverse effects and develop comprehensive practice-led training and supervision curricula for MBI instructors.

\section{Financial \& competing interests disclosure}

The authors have no relevant affiliations or financial involvement with any organization or entity with a financial interest in or financial conflict with the subject matter or materials discussed in the manuscript. This includes employment, consultancies, honoraria, stock ownership or options, expert testimony, grants or patents received or pending or royalties.

No writing assistance was utilized in the production of this manuscript.

5 Richardson J. The use of randomized control trials in complementary therapies: exploring the issues. J. Adv. Nurs. 32, 398-406 (2000).

6 Perez-De-Albeniz A, Holmes J. Meditation: concepts, effects and uses in therapy. Int. J. Psychother. 5, 49-59 (2000).

7 Yorston G. Mania precipitated by meditation: a case report and literature review. Ment. Health Relig. Cult. 4, 209-213 (2001).

8 Shonin E, Van Gordon W, Griffiths MD. Do mindfulnessbased therapies have a role in the treatment of psychosis? Aust. NZ J. Psychiatr. 48, 124-127 (2014). 
9 Sethi S, Subhash C. Relationship of meditation and psychosis: case studies. Aust. NZ J. Psychiatry 37, 382 (2003).

10 Shonin E, Van Gordon W, Griffiths MD. Cognitive behavioral therapy (CBT) and meditation awareness training (MAT) for the treatment of co-occurring schizophrenia with pathological gambling: a case study. Int. J. Ment. Health Addict. 12(2), 181-196 (2014).

11 Shonin E, Van Gordon W, Griffiths MD. Meditation awareness training (MAT) for improved psychological well-being: a qualitative examination of participant experiences. J. Relig. Health 53(3), 849-863 (2013).

12 Crane RS, Eames C, Kuyken W et al. Development and validation of the mindfulness-based interventions teaching assessment criteria (MBI:TAC). Assessment 20(6), 681-688 (2013)

13 Shonin E, Van Gordon W, Griffiths MD. Buddhist philosophy for the treatment of problem gambling. J. Behavior Addict. 2, 63-71 (2013).
14 Tsong-kha-pa. The Great Treatise On The Stages Of The Path To Enlightenment, Volume I. Cutler J, Newland G (Eds). Snow Lion Publications, NY, USA (2000).

15 Cutting Through Spiritual Materialism. Trungpa C (Ed.) Shambala, Boston, MA, USA (2002).

16 Chiesa A, Serretti A. Mindfulness based cognitive therapy for psychiatric disorders: a systematic review and meta-analysis. Psychiatry Res. 187, 441-453 (2011).

17 Hofmann SG, Sawyer AT, Witt AA, Oh D. The effect of mindfulness-based therapy on anxiety and depression: a meta-analytic review. J. Consult. Clin. Psychol. 78, 169-183 (2010).

18 Dobkin PL, Irving JA, Amar S. For whom may participation in a mindfulness-based stress reduction program be contraindicated? Mindfulness 3, 44-50 (2012).

19 Van Gordon W, Shonin E, Sumich A, Sundin E, Griffiths MD. Meditation awareness training (MAT) for psychological well-being in a sub-clinical sample of university students: a controlled pilot study. Mindfulness 5(4), 381-391 (2014). 
Reproduced with permission of the copyright owner. Further reproduction prohibited without permission. 